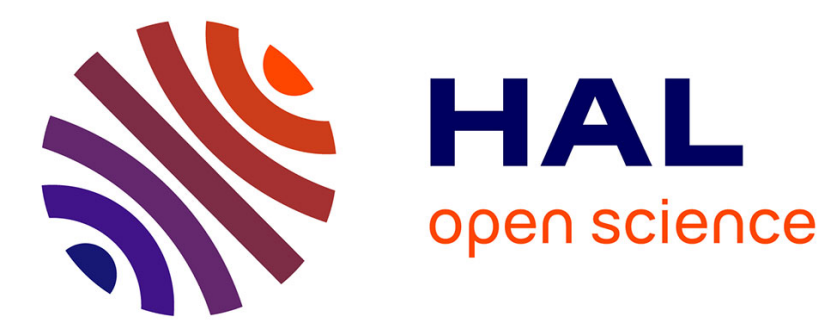

\title{
Articuler cognition spatiale et cognition environnementale pour saisir les représentations socio-cognitives de l'espace
}

Thierry Ramadier

\section{- To cite this version:}

Thierry Ramadier. Articuler cognition spatiale et cognition environnementale pour saisir les représentations socio-cognitives de l'espace. Revue Internationale de Géomatique, 2020. halshs-03286550

\section{HAL Id: halshs-03286550 \\ https://shs.hal.science/halshs-03286550}

Submitted on 14 Jul 2021

HAL is a multi-disciplinary open access archive for the deposit and dissemination of scientific research documents, whether they are published or not. The documents may come from teaching and research institutions in France or abroad, or from public or private research centers.
L'archive ouverte pluridisciplinaire HAL, est destinée au dépôt et à la diffusion de documents scientifiques de niveau recherche, publiés ou non, émanant des établissements d'enseignement et de recherche français ou étrangers, des laboratoires publics ou privés. 


\title{
Articuler cognition spatiale et cognition environnementale pour saisir les représentations socio-cognitives de l'espace
}

\author{
Thierry Ramadier ${ }^{1}$ \\ 1. Laboratoire SAGE, CNRS/université de Strasbourg \\ 5 allée du Général Rouvillois, F-67000 Strasbourg, France
}

\begin{abstract}
RESUME. Cet article s'appuie sur un ensemble de recherches sur la cognition spatiale afin de montrer, d'une part que des résultats encore éparses plaident un faveur d'une construction sociale des "cartes mentales 》, et d'autre part que c'est en conjuguant l'analyse des significations sociales de l'espace géographique (cognition environnementale) avec celle des distributions topologiques des éléments géographiques intériorisées par les individus (cognition spatiale) qu'il est possible de rendre compte de cette socialisation de la cartographie cognitive. L'auteur interroge ainsi les raisons qui font obstacle à une analyse socio-cognitive des "cartes mentales », alors que par ailleurs les représentations cartographiques sont maintenant largement reconnues comme étant des constructions sociales. Il propose de favoriser cette articulation entre cognition spatiale et environnementale en explorant de manière interdisciplinaire l'hypothèse selon laquelle les significations sociales de l'espace servent en quelque sorte de « coordonnées géographiques » aux représentations cognitives de l'espace.
\end{abstract}

MOTS-CLES : représentations spatiales, représentations sociales, cognition spatiale, cognition environnementale, lisibilité sociale de l'espace, transdisciplinarité

\section{Introduction}

Quand il s'agit d'analyser le rapport des individus à leurs espaces de vie, la recherche en sciences humaines et sociale aborde les représentations de l'espace géographique de deux manières distinctes. La première consiste à relever les significations collectives associées aux lieux: les recherches portent alors sur des représentations sociales construites à partir de la cognition environnementale, c'est-à-dire à partir des qualités environnementales des lieux). La seconde consiste à recueillir la distribution spatiale de ces lieux : ce sont cette fois les représentations spatiales, construites à partir de la cognition spatiale, c'est-à-dire à partir de processus générateurs de configurations spatiales qui sont étudiés. Or, il est frappant de constater que ces deux approches sont rarement articulées. Elles tendent alors à suivre et à alimenter les lignes de partage entre les disciplines de science sociales (histoire, sociologie, géographie sociale) et celles qui se réfèrent plus volontiers aux modèles cognitifs de la psychologie (neuro-physiologie, architecture, géographie cognitive/analyse spatiale) de sorte que, premièrement elles contribuent fortement à distinguer les quatre modèles théoriques en vigueur sur la représentation cognitive de l'espace, deuxièmement, elles structurent la manière dont les modèles théoriques envisagent la relation entre pratiques et représentations, et troisièmement elles accordent une place différente aux logiques sociales dans la construction des représentations spatiales. Cet article propose néanmoins de montrer l'intérêt de saisir ensemble ces deux manières d'analyser la construction des représentations cognitives de l'espace géographique, en s'appuyant sur la dimension épistémologique que nous a apporté ce qui s'appelle dorénavant le « tournant spatial », à savoir que l'espace est relationnel, « c'est-à-dire qu'il doit être considéré non pas comme un absolu mais comme une mise en relation des entités qui peuplent le monde » (Pattaroni, 2016), et en reprenant ces trois éléments de controverse scientifique dans le détail. Ce déroulé est guidé par une question et une hypothèse. Alors que les représentations cartographiques sont maintenant largement reconnues comme des constructions sociales (Harley, 1989), pourquoi les relations spatiales entre les objets géographiques mémorisés par les individus peinent-elles à être également étudiées de cette manière ? Un des moyens d'étudier sous cet angle les représentations cognitives de l'espace consiste à explorer l'hypothèse générale selon laquelle les significations sociales de l'espace jouent le rôle de « coordonnées géographiques » dans l'élaboration des représentations spatiales intériorisées.

\section{Cognition spatiale, cognition environnementale}

Toutefois des prolégomènes terminologiques s'imposent quand la recherche s'inscrit dans un contexte interdisciplinaire, voire transdisciplinaire (Ramadier, 2004). En effet, c'est depuis la psychologie environnementale, et avec le souci de la pratiquer en tenant compte des points de vues sociologiques et géographiques, que seront abordées les représentations de l'espace géographique. En conséquence, nous distinguerons tout d'abord la représentation cartographique de la «représentation spatiale » (figure 1) pour 
réserver la première aux cartes topographiques érigées par le cartographe, alors que la seconde concerne les configurations spatiales telles que les individus les intériorisent ${ }^{1}$. Toutefois, les représentations spatiales sont soit envisagées comme des «cartes mentales » $»^{2}$ quand elles sont conçues comme des images cognitives subjectives, individuelles et expérientielles de l'espace géographique. Quand c'est la construction sociale de ces images mentales qui est mise en avant, elles sont dénommées « représentations socio-spatiales » (Jodelet, 1982). Ce niveau de distinction sémantique permet de s'affranchir d'un trop fort tropisme mentaliste (la carte «mentale ») qui restreint le rapport à l'espace à un écheveau d'expériences personnelles plus ou moins contraintes et en lien étroit avec des préférences toutes aussi personnelles.

\section{[insérer figure 1 ici]}

La notion de représentation socio-spatiale contient encore une dernière incertitude quant à la composition de la représentation de l'espace géographique. En effet, elle peut regrouper des constructions sociales qui conservent effectivement de l'information spatiale, et on la nomme alors « représentation socio-cognitive de l'espace ». Mais cette terminologie peut aussi concerner des « représentations sociales de l'espace » qui sont exempts d'information spatiale entre les objets, autrement dit se référer uniquement à un système de significations sociales associées à l'espace géographique.

Ces distinctions permettent de conserver à l'esprit deux types de relations entre les objets géographiques. Il y a d'une part les relations basées sur des distinctions en termes de valeurs, de croyances, d'opinions, d'attitudes (ex : ce quartier est plus animé que les autres; je me sens mieux ici que là-bas; cette place est plus verte que l'autre ; etc.), qui peuvent également être perçues comme des caractéristiques absolues de l'objet géographique (respectivement : c'est un quartier animé ; c'est un lieu sûr, cette place est verte). Il y a d'autre part les relations basées sur des configurations spatiales (ex : ce quartier est plus loin du centre que l'autre; ce magasin est de ce côté de la place, etc.), qui peuvent aussi être perçus comme des caractéristiques propres à l'objet (respectivement : c'est un quartier à l'écart ; c'est le magasin du quartier).

Ces précisions terminologiques permettent également de problématiser la représentation socio-cognitive de l'espace comme le révélateur d'un rapport à l'espace géographique et social, et d'éviter par la même occasion de l'envisager comme un état mental de l'individu. La notion de rapport à l'espace peut paraître vague. Elle traduit tout simplement la manière de faire avec l'environnement (au sens d'environs géographiques ${ }^{3}$ ). Or ce rapport est multidimensionnel (Ittelson, 1973 ; Ramadier, 2009). Il peut par exemple reposer sur une dimension fonctionnelle : optimiser le choix d'un lieu de destination pour sa distance, son coût sur place ; privilégier un lieu connu, etc. Il peut aussi porter sur des dimensions plus sociales : chercher à se sentir en sécurité, à l'aise ou à sa place. Enfin, il peut avoir une dimension symbolique: chercher l'authenticité d'un lieu, une esthétique architecturale ou urbanistique particulière, un trajet ou un lieu à forte charge symbolique comme faire le chemin de Saint-Jacques de Compostelle. A l'échelle de la personne, ces dimensions ne sont pas exclusives. La représentation sociocognitive de l'espace contribue à faire ressortir soit la dimension dominante soit une modalité spécifique de la dimension : le coût plutôt que le temps dans le rapport fonctionnel par exemple. Elle permet surtout de saisir les enjeux sociaux associés à chaque groupe social. Par exemple, en décembre, les strasbourgeois qui résident au centre-ville ont tendance à mettre en avant un rapport fonctionnel à leur quartier, en insistant sur le fait qu'il est difficile de se déplacer quand les touristes arrivent en masse sur les lieux lors du marché de Noël. En revanche, les touristes apprécient l'animation des rues bondées, et les résidents des alentours fréquentent avec fierté un espace auquel ils s'identifient d'autant plus qu'il est prisé au-delà de la ville. En conséquence, le rapport à l'espace est une pratique spatiale qui se déploie à la fois en acte et en représentation.

Surtout, cette distinction entre représentations socio-cognitives et représentations sociales de l'espace renvoie à deux types de processus cognitifs : d'une part la cognition environnementale et d'autre part la cognition spatiale. Conformément aux précisions terminologiques qui viennent d'être faites, la cognition environnementale correspond à la construction sociale de croyances, opinions et significations de l'espace géographique organisées

1 Nous n'aborderons pas ici les représentations picturales de l'espace (photos, films, tableaux, cartes postales, maquettes, etc.) et les représentations cartographiques ne sont évoquées que dans le but de faciliter la démonstration selon laquelle les représentations spatiales ne sont pas simplement des représentations subjectives qui s'opposeraient aux représentations objectives du cartographe, une opposition qui entrave et limite l'approche relationnelle de l'espace. Si la carte topographique est construite comme un modèle qui réplique en partie un espace géographique, et où le cartographe cherche à mettre autant qu'il le peut à distance son propre rapport à l'espace avec méthode (rechercher la position de toutes les positions), la représentation spatiale est alors un modèle qui réplique en partie les rapports socio-spatiaux.

2 On se réfère ici au terme le plus usité, notamment dans la littérature scientifique française. Pour une recension des termes employés, voir Ramadier (1997).

3 Dans cet article, l'environnement est défini à partir de l'individu, et entendu comme « un site pour l'action, composé de la matérialité géographique (milieu) en relation avec le produit symbolique des expériences individuelles et collectives (significations). » (Ramadier, 1997, p.33). Cette acception ne limite pas l'environnement à sa dimension matérielle et physique, et elle s'articule à la conception relationnelle de l'espace géographique. 
entre elles, alors que la cognition spatiale correspond à l'élaboration et à l'intériorisation des relations spatiales entre les objets géographiques.

\section{Un difficile mariage}

Si ces deux manières d'aborder les représentations spatiales sont rarement abordées ensemble, c'est que le découpage disciplinaire de la recherche et son cortège de positions épistémologiques y contribue fortement. Prenons deux exemples d'études sur les représentations de l'espace urbain dont les auteurs ont en commun de se référer au concept d'image.

Kevin Lynch (1960), architecte et urbaniste, propose dans son ouvrage « the image of the city » une analyse qui a fait date sur la manière dont les citadins organisent leur représentation de l'espace. Il précise d'emblée que ses recherches évacuent la question de significations, qu'il estime trop labiles, pour se concentrer sur la saillance cognitive des lieux à partir de cinq catégories d'objets géographiques : les voies, les limites, les nœuds, les quartiers et les points de repère. Il propose alors le concept de lisibilité pour évoquer la facilité avec laquelle un individu identifie les éléments urbains de sorte à structurer la ville en un schéma cohérent. Ainsi, il analyse indissociablement la matérialité et sa représentation et écarte alors les pratiques spatiales quotidienne qui ne sont ici que des variables de sortie envisagées à l'échelle de l'individu et que l'urbaniste se doit de faciliter.

Raymond Ledrut (1973), sociologue, propose quant à lui dans son ouvrage « les images de la ville » une toute autre analyse de ces images ${ }^{4}$. Définissant ces dernières comme « une structure du discours portant sur un objet » (p.15), il recherche alors les significations sociales récurrentes que suscite l'évocation du nom d'un lieu ou une photographie. L'organisation spatiale des éléments urbains est ici complétement éludée. Enfin, contrairement à Lynch, et conformément à l'approche sociologique, pratiques et représentations sont envisagées comme deux facettes indissociables d'un même objet alors que la matérialité est reléguée au rang d'un support.

D'autres recherches depuis lors ont tenté de s'immiscer entre le matérialisme et le symbolisme de ces deux approches. Par exemple, Denise Jodelet (1982), dans une recherche sur la représentation de Paris a montré que les significations sociales comme la richesse ou la pauvreté, le peuplement par la migration ou non, etc., reposent sur des localisations (à l'échelle des arrondissements dans cette étude) qui l'amène à formuler l'équation selon laquelle les représentations spatiales sont des représentations sociales. Néanmoins, c'est toujours le contenu (social et socialement consensuel cette fois) de la catégorie spatiale qui produiraient les significations, et non les significations qui construisent la catégorie spatiale et la positionne topologiquement par rapport à d'autres. Autrement dit, on retrouve ici le principe des cartes thématiques de la géographie sociale, auquel est associé l'observation d'un consensus social (alors que la carte thématique repose notamment sur des récurrences, souvent statistiques). Et quand ce sont les enjeux sociaux du rapport à l'espace qui sont étudiés, il n'est à nouveau plus question de structures spatiales intériorisées, mais de conflits sociaux, d'appropriation collective et d'histoire sociale des lieux ${ }^{5}$. Tout se passe comme si ces rapports sociaux ne reposaient sur aucune configuration spatiale des catégories géographiques qui la composent. Les approches culturalistes de la cartographie cognitive (Heft, 2013) relèguent les dimensions sociales à des processus normatifs ou à des «styles cognitifs » (Evans, 1980) qui caractérisent des groupes sociaux et qui agissent comme des «filtres » (Cauvin, 1999) sur les processus cognitifs, ce qui écarte en conséquence de l'analyse tous rapports sociaux entre groupes.

Certaines approches neuro-cognitives récentes ont pour leur part cherché à articuler la cognition spatiale à la nature du cadre de référence à partir duquel la représentation spatiale se construit (Byrne et al., 2007 ; Proulx et al., 2016, pour une synthèse). Elles ont alors mis en évidence deux processus neuro-cognitifs qui distingueraient les individus entre eux : d'un côté certaines personne construisent une image spatiale à partir d'eux et de leur position dans l'espace (cadre de référence égocentrique), quand d'autres construisent des relations spatiales à partir d'un autre objet que leur propre corps (cadre de référence spatial allocentrique). Ces travaux sont dans la lignée des premiers travaux de la psychologie différentielle sur la dépendance ou non au champ (Witkin, 1949) qui cherchaient surtout à repérer des processus bio-psychologiques fondamentaux. En revanche, le psychologue JeanPaul Codol (1985) a plus fortement montré l'interrelation entre cognition spatiale et rapports sociaux en mettant en évidence le fait que l'estimation des distances physiques entre deux individus était asymétrique et dépendait de l'origine socio-spatiale sur laquelle repose l'évaluation. En effet, il constate que les individus surestiment systématiquement la distance physique entre eux et d'autres personnes quand ils sont eux-mêmes l'origine géographique de la distance (à quelle distance êtes-vous des autres ?). A l'inverse, ils sous-estiment la distance quand ce sont les autres qui sont l'origine géographique (à quelle distance les autres sont de vous ?). Autrement dit, ici c'est la signification de la distance qui fait la distance, et non pas le «contenu » de la distance (obstacle,

4 « Rien qui puisse être considéré comme une "image mentale [...] Ceci ne pouvait conduire bien loin, guère plus qu'à "l'image mentale" d'une ville qui n'a que peu de rapport avec l'Image et les Images de la ville. » (Ledrut, 1973, p. 15) 5 Voir par exemple les récents travaux de Jodelet $(2012 ; 2013)$ sur les conflits de mémoire à Nantes. 
nombre d'objets ou d'évènements rencontrés, etc.). Ces résultats renforcent ceux que les psychologues environnementalistes ont trouvé dans les estimations de distance entre un objet géographique quelconque et un point de référence spatial (Sadalla et al., 1980), c'est-à-dire entre un élément de la cartographie cognitive qui produit, résume et représente une catégorie spatiale et un élément urbain quelconque de cette catégorie spatiale. Par exemple, la gare est le point de référence du quartier gare dans la mesure où elle participe au découpage de la ville en produisant ce quartier et en le représentant symboliquement. Or il s'avère que toutes les estimations de distance au départ de la gare vers d'autres entités du quartier gare sont plus courtes que dans le sens inverse (quand l'estimation s'effecute au départ d'une entité quelconque du quartier). Ce sont là les premiers éléments d'une influence des significations socio-spatiales sur les configurations spatiales. Une autre étude plus récente a montré que le concept de lisibilité de Kevin Lynch (1960) avait également une composante sociologique : malgré le même temps passé sur les lieux, les groupes sociaux qui étaient familiers avec les signes et les codes de la ville attribuaient du sens aux éléments de leur représentation sur de plus nombreuses dimensions de l'espace (dimensions physiques, sociale, fonctionnelle et d'usage) que ceux qui étaient moins familiers (essentiellement sur la dimension d'usage), et la structure spatiale de leur représentation s'en trouvait fortement affectée (Ramadier et Moser, 1998). Preuve que cette habituelle dissociation entre cognition spatiale et environnementale n'est pas incontournable !

\section{Stimulation, perception, représentation}

Pour tenir ensemble la matérialité de l'espace géographique, ses dimensions symboliques et les rapports sociaux liés à ces dernières, il importe tout d'abord de replacer la représentation dans son contexte socio-cognitif. En effet, comprendre les différences entre stimulation, perception et représentation permet de préciser ce que cette dernière doit aux rapports sociaux.

Le premier niveau de notre rapport au monde repose sur la stimulation. Cette activité biophysicaliste n'est pas consciente, et fort heureusement, car cela permet de ne pas se noyer dans la complexité et le foisonnement des stimuli que nous impose chaque situation géographique. C'est d'ailleurs une des raisons qui fait que l'environnement n'agit pas en soi sur l'individu. Tant que la stimulation n'a pas d'importance pour notre engagement au monde, elle a peu d'emprise sur notre mémoire, nos évaluations, nos significations (figure 2).

\section{[insérer la figure 2 ici]}

En revanche, dès que la stimulation devient une information, autrement dit dès lors qu'elle est chargée de sens, ce sont des processus perceptifs qui sont en jeu et qui font intervenir notre mémoire, nos croyances, notre jugement pour nous permettent d'appréhender où l'on est, qui est là, si nous sommes proches ou loin du lieu que nous cherchons à atteindre, etc. L'enjeu de la perception est alors situationnel, et la situation est à la fois spatiale (la localisation et l'orientation du corps dans l'espace), psychologique (dépendante de schèmes de perception intériorisés au cours de l'histoire géographique de l'individu ${ }^{6}$ ), sociologique (dépendante de schèmes de perception intériorisée lors du processus de socialisation à l'espace ${ }^{7}$ ). L'information est nécessairement socialisée. Et dans le même temps, elle est situationnelle, c'est-à-dire que le corps est nécessairement concerné, ce qui fait que la stimulation est toujours présente dès qu'il s'agit de perception (figure 2). En un mot, la perception est dépendante d'un double système, car elle s'élabore autant à partir de processus biologiques que sociologiques.

La représentation, quant à elle, n'est plus qu'information reconstruite à partir de traces mémorielles, de croyances, d'évaluations. Elle est centrée sur les significations qui, une fois de plus, sont des constructions sociales. Par exemple Valérie Haas (2002) s'est appuyée sur les travaux de Maurice Halbwachs (1950) sur la mémoire collective pour saisir les particularités de la représentation de Vichy auprès de ses résidents. Elle constate alors que le siège du gouvernement d'occupation («gouvernement Pétain») n'était jamais mentionné dans les représentations spatiales alors que le bâtiment était toujours reconnu quand elle présentait sa photo aux enquêtés. Les enjeux de la représentation ne sont plus situationnels, ils sont avant tout symboliques, et par conséquent sociaux (Dubois et al., 2005). D’un point de vue géographique, ces enjeux ne reposent pas sur des localisations uniques et absolues, mais sur des positions relatives entre objets, une proposition très tôt énoncée par Vidal de la Blache (1902) pour comprendre les phénomènes géographiques. C'est d'ailleurs le cas pour toutes représentations.

\footnotetext{
6 Cette dimension, proche de la notion d'expérience, concerne surtout les pratiques et des dispositions mentales qui sont transposables d'un espace à l'autre et qui s'apparentent à des «affordances » (Gibson, 1979), ou mieux, à des «sites comportementaux »(Barker, 1968). C'est, par exemple, la suite des interactions avec le personnel et des comportements quand on arrive dans un restaurant, qui ne sera toutefois pas la même que quand on arrive dans un snack, bien que nous n'ayons encore jamais fréquenté chacun des lieux.

7 Cette dimension englobe, mais aussi dépasse les seules spécificités culturelles du rapport à l'espace. En effet, elle repose également sur des différences qui s'inscrivent dans des rapports sociaux, comme des différences de genre (les femmes ne perçoivent pas de la même manière que les hommes la situation environnementale d'une promenade urbaine nocturne par exemple), de classe sociale (se sentir à sa place), etc. Sur ce point, voir Rivière (2017).
} 
La représentation cartographique n’y échappe pas! Par exemple, entre un planisphère nord-américain, asiatique et européen (figure 3), les informations topographiques restent les mêmes. En revanche, les informations topologiques, c'est-à-dire les significations symboliques associées aux relations spatiales (central ou périphérique, en haut ou en bas) peuvent fortement varier. Ainsi, malgré la construction mathématique des planisphères, les enjeux de centralités ou de décisions à propos des distorsions spatiales, bref de présentation de soi et des siens, renvoient à des dimensions autant symboliques que fonctionnelles de l'image produite. Les travaux de Saarinen (1987) sur les représentations cognitives du monde, auprès de 3863 personnes réparties dans 41 pays sur l'ensemble du globe, retrouvent ces trois mêmes types de topologie mondiale (figure 4), avec des variations qui dépendent effectivement de l'aire géographique de résidence des enquêtés (Amérique, Europe, Asie).

\section{[insérer figure 3 et 4 ici]}

Ce n'est donc pas l'opposition entre subjectivité et objectivité qui distingue représentations spatiale et représentation cartographique. Dès lors, la représentation cartographique n'est pas une référence absolue. Elle reste une référence utile parce que l'on connaît exactement son processus de construction. Mais la prendre comme référence absolue, c'est prendre le risque de raisonner en termes d'erreurs géographiques, ce qui n'apporte en soi pas grand-chose à la compréhension des rapports à l'espace. Prenons un exemple simple : si on se pose la question de la distance qui sépare Paris de Lyon, nous ne recueillerons pas la même réponse ni la même mesure, selon qu'on la pense ou qu'on la mesure à vol d'oiseau, par la nationale, par l'autoroute ou par le train. On ne peut définir une distance réelle qu'à partir du moment où l'on définit un mode de déplacement. Or ce mode de déplacement dit déjà quelque chose sur le rapport à l'espace, et plus généralement sur les modes de vie.

D'ailleurs, les logiciels d'aide à la construction d'un itinéraire sur internet ne s'y trompent pas, car leurs algorithmes proposent une réponse en fonction de différentes modalités du rapport fonctionnel au déplacement : le moins cher, le plus court, le plus rapide. Autrement dit, le traitement systématique par des mesures connues et explicites fait de la carte géographique un référentiel technique qui ne veut pas forcément dire quelque chose à tout le monde, mais qui permet au chercheur de mesurer l'importance des surestimations ou des sous-estimations de distances. Cependant, que nous apporte une telle mesure d'un point de vue sociocognitif ? Car pour comprendre les rapports à l'espace, on peut se contenter d'objectiver des écarts entre groupes sociaux en calculant puis en comparant leurs estimations moyennes. Néanmoins, la carte géographique est particulièrement utile comme outil pour objectiver les distorsions sur les formes spatiales signifiantes. Par exemple, si la courbure de la Seine à Paris est généralement simplifiée (aplatie), c'est que le fleuve sert surtout à découper la ville en deux (Milgram et Jodelet, 1976). Le cours d'eau devient un axe qui structure mentalement et matériellement toute l'organisation fonctionnelle et symbolique de la ville. L'aplatissement du cours d'eau pose plus de problèmes aux enquêtés pour positionner le $16^{\text {eme }}$ arrondissement que pour s'y rendre ou lui attribuer un ensemble de significations partagées. Autrement dit, en termes d'investigation sur les représentations sociocognitives, la carte géographique suppose un usage raisonné, plutôt que de l'envisager d'emblée comme la référence géographique objective. En effet, de même qu'il est maintenant notoire qu'une représentation cartographique dépend des enjeux sociaux qui lui sont associés (Harley, 1989), il en est de même pour les représentations spatiales. Leur élaboration repose sur la position sociale de celui ou de ceux qui l'érigent (Dias et Ramadier, 2015 ; 2018). Comment se fait-il que les enjeux sociaux des représentations cartographiques sont maintenant largement reconnus alors que ceux des représentations spatiales peinent à l'être ? Probablement parce que les significations qui sont au fondement des représentations sociales de l'espace sont également largement chargées d'enjeux sociaux qui restent néanmoins dissociées des configurations spatiales intériorisées.

\section{Quatre familles de modèles théoriques sur les représentations spatiales}

Les différents modèles théoriques en cours sur les représentations spatiales sont révélateurs de cette difficulté à introduire les rapports sociaux et les représentations sociales dans l'analyse de leur configuration spatiale. Robert Kitchin (1994) dégage quatre types de modèles dans la littérature scientifique sur la cognition spatiale.

Le premier correspond au modèle littéral de la carte mentale ${ }^{8}$. Il est essentiellement porté par les neurosciences qui ont par exemple mis en évidence des « cellules de lieu » (O'Keefe et Dostrovsky, 1971). Ce sont des cellules qui s'activent quand l'individu, jusque-là des souris, passe à un endroit précis du terrain d'expérimentation. La carte mentale serait alors constituée d'un réseau de neurones élaboré à partir d'expériences géographiques directes ou secondaires. Ce modèle néo-behavioriste des représentations envisage la carte mentale comme une carte transposée dans un réseau de neurones.

\footnotetext{
${ }^{8}$ Ici l'expression carte mentale est sans guillemets, afin de souligner l'approche mentaliste de ce modèle, une position épistémologique sur laquelle se réfère les chercheurs qui le développe.
} 
Le second modèle correspond à la «carte mentale » fonctionnelle. Il s'appuie cette fois sur un double codage de l'information disponible en mémoire (Pavio, 1974) : l'information géographique est d'abord stockée en mémoire sous la forme de concepts. Ils sont ensuite utilisés sous la forme d'une image mentale construite à partir de distances subjectives entre les éléments géographiques mémorisés qui sont directement en relation avec les distances objectivées par l'espace euclidien (Shepard, 2001). Les distances estimées seraient alors extraites par une sorte d'œil mental qui scannerait l'image produite. Ce modèle repose sur un postulat : plus la représentation spatiale est proche de la carte géographique et plus la première serait un outil cognitif efficient et fiable pour les pratiques quotidiennes. La représentation spatiale serait une représentation analogique de la représentation cartographique. Cette confrontation directe des représentations des individus à la représentation cartographique fait de ce modèle celui qui alimente le plus l'idée que les représentations spatiales sont des représentations subjectives.

Le troisième modèle correspond à celui de la métaphore de la «carte mentale ». Les processus cognitifs sont les mêmes que précédemment, à savoir un double codage de l'information géographique, à la différence près qu'il n’y a plus de métrique géographique. Les relations spatiales sont cette fois topologiques (en haut, plus loin que, etc.), ce qui les rapproche fortement des significations associées aux éléments représentés. Par exemple, le fait qu'un lieu soit de l'autre côté d'une voie de chemin de fer (barrière physique) ou dans une autre commune (barrière symbolique) fait qu'il va être jugé plus éloigné qu'un autre lieu situé à la même distance que le lieu de référence. On comprend ici que ce modèle est le plus adapté pour saisir simultanément les dimensions physiques, sociales et symboliques associées aux relations spatiales des éléments mémorisés.

Enfin, le quatrième modèle correspond à la représentation spatiale comme construction hypothétique. À l'opposé du premier modèle, ici la représentation spatiale n'est qu'une représentation conceptuelle. L'image mentale n'est pas convoquée et les relations spatiales représentées ne sont pas euclidiennes (Pylyshyn, 1973). Les relations représentées visuellement par le dessin ne sont que des cheminements d'associations entre les concepts géographiques représentés. Cela correspond finalement à des cartes heuristiques, c'est-à-dire à des représentations picturales qui mettent en relation des concepts, des idées, des représentations entre elles.

[Insérer la figure 5 ici]

En revenant sur ce que sont la cognition spatiale d'une part et la cognition environnementale d'autre part, on constate que le principal point de controverse entre ces quatre modèles repose sur l'articulation ou non, le mariage ou non pourrait-on dire, entre ces deux types de processus qui participent à la construction cognitive de l'espace géographique. En effet, on constate que le premier et le quatrième modèle écartent un tel mariage entre cognition spatiale et environnementale : le premier se limite à la cognition spatiale alors que le dernier se focalise sur la cognition environnementale. Pour le second et au troisième modèle, qui s'appuient sur le modèle cognitif du double codage de l'information, c'est sur la manière d'articuler ces deux processus cognitifs que la controverse porte. Le modèle analogique (modèle 2) conserve la primauté de la cognition spatiale sur la cognition environnementale, cette dernière n'ayant qu'une fonction de stockage de l'information en mémoire alors que ce sont les distances métriques euclidiennes qui constituent l'information de base des distributions spatiales des lieux intériorisés. Le modèle de la métaphore (modèle 3) accorde en revanche plus d'importance à l'évaluation environnementale, la cognition spatiale étant elle-même évaluative et construite à partir d'une topologie qui produit une métrique relationnelle (plus loin que, à côté de, à droite de, etc.).

Quelques recherches montrent, à partir des positions sociales des enquêtés, qu'une dimension sociale non négligeable intervient dans la construction des représentations spatiales. Cependant, les modèles théoriques supportent finalement difficilement un tel constat. Seul le modèle de la métaphore de la «carte mentale » (troisième modèle présenté) est le plus susceptible d'appuyer de telles recherches. Or, le modèle du double codage (Pavio, 1974) sur lequel il repose n'articule pas véritablement la cognition spatiale et environnementale, car les premiers processus restent dévolus aux pratiques spatiales (activation d'une image fonctionnelle) alors que les seconds sont réservés au stockage de l'information en mémoire. Ainsi, ce modèle psychologique reste très sommaire et n'a rencontré aucune évolution depuis lors.

\section{Représentations, pratiques et modèle relationnel}

Nous avons vu que le découpage disciplinaire ne s'avérait pas propice à la mise en correspondance de la cartographie cognitive avec les significations sociales de l'espace. Du côté des promoteurs de la «carte mentale », tout se passe comme si leurs significations étaient plus individuelles (préférences) que sociales, nourrissant et nourries par des comportements qui constituent l'expérience physique de l'espace géographique. Dès lors, les significations sociales sont des interprétations surplombantes du chercheur. L'enquêté se trouve en quelque sorte dépossédé de ses constructions, et reste cantonné aux conditions sociales de son expérience personnelle (relever par exemple les lieux évalués positivement selon le genre pour en déduire des préférences environnementales, 
plutôt que de relever les significations des lieux afin de compléter les connaissances sur les pratiques spatiales genrées). Autrement dit, la dimension symbolique des rapports et des enjeux sociaux n'a que peu de place dans les analyses sur l'élaboration des relations spatiales. Pourtant, il est maintenant établi que les sur-estimations de distances géographiques peuvent tout simplement être le fait de barrières symboliques entre les deux points (Steven et Coupe, 1978), et les résultats sont comparables à ceux observés avec des barrières physiques, comme un cours d'eau par exemple.

Chez les artisans des représentations sociales de l'espace, la position du chercheur s'inverse. Cette fois, les représentations socio-cognitives de l'espace ne sont pas aussi déterminantes pour les pratiques spatiales ... car toutes deux ne forment qu'un ensemble indissociable ${ }^{9}$ : le rapport à l'espace. Dès lors, le relevé des relations spatiales entre objets géographiques mémorisés est surtout un moyen d'objectiver des significations, non de saisir ce qu'elles permettent en termes de pratiques spatiales. Autrement dit, cette fois les enquêtés sont dépossédés de leur représentation spatiale. Celle-ci est reconstruite par le chercheur, souvent à partir d'une liste de lieux reliés entre eux par leurs occurrences ou leurs significations plutôt que par des relations spatiales (figure 5). Le modèle théorique sous-jacent est alors plus souvent celui de la représentation spatiale hypothétique » (4 ${ }^{\text {ème }}$ modèle).

\section{[insérer la figure 6 ici]}

Ainsi, au sein des partis-pris épistémologiques, c'est d'une part la place de la matérialité et d'autre part la relation entre pratiques et représentations qui sont au cœur des divergences théoriques et de la difficulté à conjuguer cognition spatiale et environnementale. Pour les faire converger, il importe alors de sortir de la dichotomie subjectivité/objectivité.

Si nous avons déjà précisé que nos représentations spatiales ne sont pas subjectives, elles ne sont pas non plus objectives. Elles sont tout autant fondées sur l'espace physique que sur les aspects symboliques que l'individu retire de cette matérialité. Elles sont tout autant fondées sur l'action du corps (comportement moteur) que sur ce que ces actions disent des relations et des enjeux sociaux. Il est dans ce cas difficile de conserver une perspective interactionniste qui sépare le sujet de l'objet, qui définit l'un indépendamment de l'autre, faisant de chacun d'eux une entité existant par elle-même. Mais il est également difficile de conserver une perspective où les pratiques spatiales peuvent être déduites des représentations spatiales, ou inversement, sous prétexte de consonance cognitive. En effet, autant le modèle interactioniste que celui qui postule les pratiques comme indissociables des représentations poussent à une telle posture centrées sur la congruence, hors des contraintes, entre pratiques et représentations. Pourtant, une recherche sur un quartier pavillonnaire de la banlieue de Strasbourg (Ramadier et al., 2008), nous a permis de montrer qu'il n'y avait pas nécessairement de congruence entre pratiques et représentations de la ville : bien que les moins diplômés du quartier allaient peu au centre-ville, notamment parce que leur lieu de travail était en banlieue ou hors de l'agglomération, la part des éléments du centre-ville dans leurs représentations de Strasbourg était plus importante que celle des plus diplômés qui travaillaient et fréquentaient plus souvent le centre-ville.

Sortir de la perspective interactionniste permet de se focaliser sur la qualité des relations entre pratiques et représentations plutôt que sur leur cohérence géographique a priori. Aussi, dans la mesure où les représentations sont envisagées comme un indicateur socio-cognitif du rapport à l'espace, une perspective relationnelle (figure 6), que l'on trouve dans la littérature de psychologie environnementale sous le nom de perspective transactionnelle (Altman et Rogoff, 1987), constitue un puissant moyen de conjuguer cognition spatiale et sociale. Cette approche relationnelle permet de privilégier une analyse centrée sur la correspondance (plutôt que sur la cohérence $a$ priori) entre les dimensions sociales et les dimensions psychologiques de l'activité cognitive. On retrouve alors ici à l'échelle de l'individu ce que Durkheim et Mauss (1905) montraient à l'échelle des rapports sociaux, à savoir une correspondance entre les structures sociales et les structures cognitives. Enfin, elle permet également d'accorder une place à la matérialité de l'espace géographique qui n'est pas qu'un simple support pour les rapports sociaux. En effet, comme le propose Jean Remy (2015), la matérialité médiatise plus qu'elle ne supporte les rapports sociaux, ce qui renforce par ailleurs la proposition d'analyser des correspondances plutôt que des cohérences entre pratiques et représentations.

[insérer la figure 7 ici]

\section{Objet géographique, catégorie spatiale}

L'approche relationnelle a des implications importantes sur la manière d'analyser les représentations sociocognitives de l'espace. Elle suppose de s'affranchir de l'idée que ces représentations sont constituées d'objets

9 Pour revenir à la position de Ledrut (1973) et illustrer cet aspect indissociable des représentations et des pratiques, l'auteur précise que « les significations ne sont ni dans la tête ni dans les choses, elles sont dans l'expérience : ici l'expérience urbaine » (p.12). 
géographiques qui existent en et pour eux-mêmes, et qu'ensuite ce ne serait que le résultat de leur combinaison qui prouverait que l'image de la ville est socialement construite. L'analyse par correspondance, plutôt que par cohérence, de l'approche relationnelle suppose au contraire d'aborder chaque objet de la représentation comme une catégorie spatiale, c'est-à-dire également comme des représentations sociales de l'espace. Ainsi, quand deux personnes évoquent un même «objet» géographique dans leur représentation spatiale, qui sont par ailleurs différentes, cet « objet», pourtant semblable matériellement et géographiquement, ne l'est pas socialement, et $a$ fortiori au regard des pratiques.

Envisager un élément géographique comme une catégorie plutôt que comme un objet est une raison supplémentaire d'accorder de l'importance à l'articulation entre cognition spatiale et environnementale. D'ailleurs, comme le précise Jean Laponce (2001) «il ne nous est pas possible (à moins de troubles graves) de ne pas utiliser des catégories spatiales pour nous situer dans notre environnement physique ». C'est le sens attribué à ces catégories qui donne une existence cognitive et une consistance géographique aux éléments perçus ou représentés. Pour prendre un exemple qui reste proche de la géographie, on peut avoir une attitude contemplative devant le ciel étoilé. Néanmoins, pour partager nos observations à propos d'une étoile, d'une planète ou d'un satellite perçu, il nous faut découper l'espace en unités signifiantes exclusives définies à partir d'alignements remarquables de points. Si l'on décrit une ligne qui serpente, c'est par opposition à une autre région où on mettra l'accent sur la forme d'un cercle, et à une autre où l'on s'appuiera sur une figure rectangulaire, etc. On constitue alors des contrées ${ }^{10}$ qui seront nommables et positionnables. Ainsi armé d'un système de significations environnementales du ciel, on peut évoquer son arrangement spatial et même le cartographier. Aussi, nous faisons la même chose sur terre, même si les informations sont plus denses du fait de stimulations plus variées. Autrement dit, le découpage d'un espace géographique repose sur des éléments géographiques qui ne sont jamais des objets géographiques en soi, mais des catégories spatiales construites à partir des significations environnementales qui sont associées à ces éléments ou groupes d'éléments. La remarque qui suit sur les récurrences constatées dans les représentations sociocognitives de l'espace vient compléter cette difficile distinction entre catégorie spatiale et objet géographique. Lors du relevé des représentations sociocognitives de l'espace de vie, il y a d'un côté les enquêtés qui placent leur logement dans la représentation spatiale qu'ils nous livrent, et de l'autre ceux qui ne le mentionnent pas. En termes d'objet, chaque logement est un élément géographique différent, que ce soit du point de vue de sa localisation ou en tant qu'élément physique. Mais cette idiosyncrasie n'en est plus une dès lors que l'on considère le logement comme un élément qui renvoie à une même définition ainsi qu'à une pratique sociale très proche pour chaque enquêté. On peut alors analyser la représentation en fonction de la présence ou non de cet élément, comme on le fait habituellement pour la gare, la mairie, etc. En accordant le statut de catégorie spatiale au logement (une catégorie certes pas tout à fait comme les autres éléments), on ne simplifie et n'uniformise pas pour autant le rapport des individus à leur logement. De même, on ne considère pas que le rapport à la mairie ou à la gare est le même pour tous ceux qui le signalent dans leur représentation. On se donne un moyen méthodologique de l'inclure dans l'analyse du rapport à l'espace. En revanche, si on le traite comme un objet idiosyncrasique, son analyse avec l'ensemble des autres items de la représentation est très réduite.

La notion de catégorie permet également de traiter certains éléments de la représentation autrement qu'à partir de référentiels exogènes (une définition administrative, centrée sur la localisation, etc.) pour cerner les «bonnes » ou les «mauvaises » réponses des enquêtés (par rapport à la définition exogène). Au contraire, cela permet de fonder les analyses sur des comparaisons endogènes au sein de l'échantillon interrogé. À titre d'exemple, une recherche menée sur l'agglomération de Québec (Ramadier, 2002) a permis de cerner que les limites ${ }^{11}$ du quartier de résidence provenaient finalement du rapport social, spatial et psychologique que la personne entretenait avec son quartier, mais aussi plus largement avec la ville. En interrogeant des résidents d'un quartier pavillonnaire de la banlieue, un premier constat s'est imposé : les enquêtés les plus âgés, qui avaient fait construire leur maison dans le quartier à la fin des années 50, se différenciaient de ceux qui avaient racheté une maison dans le quartier en moyenne 10 ans avant l'enquête. La définition générique de la banlieue était le seul point sur lequel les deux groupes s'accordaient. Malgré cela, les « pionniers » répondaient que leur quartier était situé en banlieue alors que la seconde génération de résidents estimait qu'ils étaient au centre géographique de l'agglomération, c'est-à-dire près de toutes les aménités urbaines. Les représentations spatiales différaient également : les premiers arrivants avaient une représentation concentrique de la ville allant du centre-ville à la périphérie alors que les plus jeunes avaient une représentation multipolaire de l'espace urbain. Enfin, en termes de pratiques spatiales, les « pionniers » structuraient leurs mobilités quotidiennes autour de la consommation, cherchant partout dans l'agglomération ce dont ils avaient besoin chez eux : bricolage, jardinage, alimentation, etc. On peut dire que leurs déplacements étaient centripètes. Par ailleurs, leur réseau de relations se situait essentiellement dans le quartier de résidence. À l'inverse, la seconde génération avait des déplacements centrifuges, dans la mesure où leurs activités étaient

10 «Son étymologie [de la contrée] est intéressante : il désigne précisément un espace différent. Il vient de contra, qui est à la fois op-posé et im-médiat, posé en face et sans médiation : contre, mais tout contre. » (Brunet, 2001, p. 119)

$11 \mathrm{Au}$ sens d'élément constitutif des représentations spatiales proposé par Lynch (1960) 
dispersées sur l'ensemble de l'agglomération : aller au restaurant ici, visiter des amis là, faire du sport ailleurs. Seule la consommation courante était faite dans la supérette du quartier. Et pour finir, d'un point de vue psychologique, l'attachement au lieu des pionniers était plus souvent limité à leur quartier, qu'ils circonscrivaient exclusivement au secteur pavillonnaire, alors que la seconde génération exprimait un attachement à l'agglomération dans son ensemble, avec des limites du quartier qui s'étendait jusqu'au secteur des logements sociaux qui se situaient de l'autre côté de la route d'accès au quartier. En outre, il s'avère que les différentes générations de résidents ne peuvent pas être réduites à leur cycle de vie ou à leur âge, car on retrouvait le profil du rapport à l'espace des pionniers auprès de populations qui avaient le même âge que la seconde génération de résidents mais qui logeaient aux franges de l'agglomération, c'est-à-dire dans des espaces où ils étaient également les «pionniers » de leur quartier résidentiel! Par conséquent, ce sont bien plus des rapports au quartier et à l'agglomération très différents qu'un effet générationnel des résidents qui expliquent les différences constatées en termes de limite géographique du quartier résidentiel. Ces résultats montrent également que l'espace géographique n'a pas de signification en soi, tout comme un élément géographique n'a pas d'existence en soi. Deux points importants pour saisir, analyser et comprendre la place des représentations dans le rapport à l'espace des individus. Enfin, cet exemple sur la localisation et la composition des limites d'une catégorie spatiale comme le quartier de résidence montre une fois de plus que les significations sociales des éléments de la représentation spatiale jouent le rôle de « coordonnées géographiques », ou plus exactement participent activement à la construction des relations topologiques et des configurations spatiales de la représentation.

\section{Pour conclure}

Les observations sur le rôle des significations sociales dans l'élaborations de la structure spatiale d'une représentation socio-cognitive de l'espace géographique sont encore très disparates : ici la localisation de limites géographiques, là des sur-estimations ou des asymétries sur les distances géographiques, ailleurs l'importance de la mémoire collective sur le contenu géographique de la représentation et enfin les dimensions sociales de la lecture de l'espace urbain. Le manque d'unité vient, comme on a cherché à la montrer ici, de la difficile articulation entre cognition spatiale et cognition environnementale.

Conjuguer étroitement cognition spatiale et cognition environnementale est un véritable travail transdisciplinaire (Ramadier, 2004) sur l'analyse du rapport des individus à leur espace de vie. Il suppose de confronter plutôt que de fusionner ou de juxtaposer les connaissances acquises par les différentes disciplines. Cela suppose par exemple de reconnaître que la sociologie rend plus efficacement compte des conditions de possibilité de la cognition spatiale : c'est elle qui est la plus à même de tenir compte de ce qui est envisageable pour la personne (l'univers des possibles) plutôt que de partir du principe que tout le monde est égal face à l'information géographique disponible. Cela nécessite ensuite de reconnaître que la psychologie a surtout développé des connaissances permettant de rendre compte des conditions de production de la cognition spatiale. Par exemple, quelques structures mentales des représentations spatiales ont été mises en évidence, comme la construction des catégories spatiales à partir des points de référence (Sadalla et al., 1980) et leur organisation en arbre hiérarchique (Hirtle et Jonides, 1985) Enfin, la géographie rend plus facilement compte des conditions de fonctionnement de la cognition spatiale. Elle a notamment développé des outils statistiques et informatiques facilitant l'analyse des relations entre représentations et pratiques spatiales. Autrement dit, l'idée n'est pas que chaque discipline traite son objet de prédilection (les rapports sociaux, les processus cognitifs, l'espace géographique), mais que toutes convergent vers la cognition spatiale à partir des diverses conditions nécessaires pour comprendre son processus et son résultat.

Si les exemples disciplinaires sont limités à la confrontation des connaissances en géographie, sociologie et psychologie, cette approche sociocognitive ne se limite pas pour autant à ces trois disciplines. L'histoire (Laboulay, 2004), la science politique (Breux et al., 2010), la sémiologie, l'économie, etc., pour ne citer que les sciences humaines et sociales, peuvent également confronter leurs modèles et leurs méthodes à partir de cette proposition. Il serait toutefois intéressant de rechercher du côté d'autres disciplines, comme les mathématiques, l'informatique ou les neurosciences, dès lors qu'elles sont à même d'objectiver des modulations spatiales et de s'appuyer alors sur le fait qu'il n'y a pas un, mais des espaces géographiques.

\section{Bibliographie}

Altman I., Rogoff B. (1987). World views in psychology: trait, interactional, organismic, and transactional perspectives. In D. Stokols \& I. Altman (Eds.), Handbook of environmental psychology, New York: Wiley, p. 7-40.

Barker R. (1968). Ecological psychology: concepts and methods for studying the environment of human behavior, Standford, Standford University Press. 
Breux S., Reuchamps M., Loiseau H. (2010). Apports et potentialités de l'utilisation de la carte mentale, en science politique, transéo review, en ligne: https://orbi.uliege.be/bitstream/2268/28605/2/Breux\%20S\%2C\%20et\%20al\%2C\%20Apports\%20et\%20potentialit\%C3\%A9s \%20de $\% 201 \% 27$ utilisation $\% 20 \mathrm{de} \% 201 \mathrm{a} \% 20$ carte $\% 20$ mentale $\% 20 \mathrm{en} \% 20$ science $\% 20$ politique $\% 2 \mathrm{C} \% 202010$.pdf

Brunet R. (2001). Le déchiffrement du monde, Paris, Belin.

Byrne P., Becker S., Burgess N. (2007). Remembering the past and imagining the future: a neural model of spatial memory and imagery, Psychological review, $\mathrm{n}^{\circ} 114, \mathrm{p} .340-375$.

Cauvin C. (1999). Pour une approche de la cognition spatiale intra-urbaine, Cybergeo : European Journal of Geography [En ligne], Politique, Culture, Représentations, document 72.

Codol J-P. (1985). L'estimation des distances physiques entre les personnes : suis-je aussi loin de vous que vous l'êtes de moi, L'année psychologique, vol. 85, n 4 , p. 517-534.

Dias P., Ramadier T. (2015). Social trajectory and socio-spatial representation of urban space: the relation between social and cognitive structure, Journal of Environmental Psychology, ${ }^{\circ}{ }^{41}$, p. 135-144.

Dias P., Ramadier T. (2017). Relations sociales et cartographie cognitive. Les points de référence comme noyau central des représentations spatiales, Cahiers internationaux de psychologie sociale, vol. 116 n4, p.319-349.

Dias P., Ramadier T. (2018). Representational Structures as Stances: Examining Relationships to the City Under the Lens of Socio-Spatial Representations, International Review of Social Psychology, vol. 31, n¹, p. 1-13.

Downs R.M., B.D. Stea B.D. (1981). Des cartes plein la tête, Ste Hyacinthe, Edisem.

Dubois J., Durand P. Winkin Y. (2005). Le symbolique est le social, In Jacques Dubois, Pascal Durand, Yves Winkin (dir.), Le Symbolique et le Social, Liège, Editions de l'Université de Liège, p.13-28.

Durkheim E., Mauss M. (1903). De quelques formes primitives de classification, Contribution à l'étude de représentations collectives, L'Année sociologique, $\mathrm{n}^{\circ} 6$, p. 1-72.

Gibson J. (1979). An ecological approach to visual perception. Boston, Houghton Mifflin.

Haas V. (2002). Approche psychosociale d'une construction historique. Le cas vichissois, Cahiers internationaux de psychologie sociale, vol. $53, \mathrm{n}^{\circ} 1$, p. 32-45.

Halbwachs M. (1950). La mémoire collective, Paris, Albin Michel

Harley J.B. (1989). Deconstructing the map, Cartographica, vol. 26, n² 2, p. 1-20

Heft H. (2013). Environment, cognition, and culture: Reconsidering the cognitive map, Journal of Environmental Psychology, $\mathrm{n}^{\circ} 33, \mathrm{p} .14-25$.

Hirtle S., Heidorn (1993). The structure of cognitive maps: representation and process, in T. Garling and R.G. Golledge (eds) Behavior and Environment: Psychological and geographical approaches. Elsevier Science Publishers.

Hirtle S., Jonides J. (1985). Evidence of hierarchies in cognitive maps, Memory and Cognition, vol. 13, n³, p. 208-217.

Ittelson W.H. (1973). Environment perception and contemporary perceptual theory, in W. H. Ittelson (Ed.), Environment and cognition, New York: Seminar Press, p. 1-19.

Jodelet D. (1982). Les représentations socio-spatiales de la ville, In P.H. Derycke (ed.), conception de l'espace, Paris, Université de Paris X, p. 145-177.

Jodelet D. (2012). Conflits entre histoire mémorielle et histoire historienne, Psicologia e Saber Social, vol. 1, n 2, p. $151-162$.

Jodelet D. (2013). Les inscriptions spatiales des conflits de mémoire, Psicologia e Saber Social, vol 2, n 1, p. 5-16

Kitchin R. M. (1994) Cognitive maps: What are they and why study them? Journal of Environmental Psychology, ${ }^{\circ} 14$, p.119.

Laboulay-Lesage I. (2004). Combler les blancs de la carte. Modalités et enjeux de la construction des savoirs géographiques (XVIIe - XXe siècle), Strasbourg, Presses Universitaires de Strasbourg.

Laponce J. (2001). Le centre du monde : icône ou carrefour ? Revue internationale de sociologie, vol. 11, n ${ }^{\circ}$ 3, p. $299-307$.

Ledrut R. (1973). Les images de la ville, Paris, Anthropos, 1973.

Lynch K. (1960). The image of the city, Cambridge, Mass.: The MIT Press.

Milgram S., Jodelet D. (1976). Psychological maps of Paris, In Harold Proshansky et al. (Eds) Environmental Psychology (2nd edition) New York, holt, Rinehart \& Winston.

O'Keefe J., Dostrovsky J. (1971). The hippocampus as a spatial map: Preliminary evidence from unit activity in the freelymoving rat. Brain Research, $\mathrm{n}^{\circ}$ 34, p. 171-175.

Pavio A. (1974). Imagery and verbal processes, New York, Holt, Rinehart \& Winston. 
Proulx M.J., Todorov O.S., Taylor Aiken A., de Sousa A.A. (2016). Where am I? Who am I? The Relation Between Spatial Cognition, Social Cognition and Individual Differences in the Built Environment, Frontiers in Psychology, n ${ }^{\circ}$, article 64 (en ligne).

Pylyshyn Z. (1973). What the mind's eye tells the mind's brain: A critical of mental imagery, Psychological Bulletin, $\mathrm{n}^{\circ} 80, \mathrm{p}$. $1-24$.

Ramadier T. (1997). Construction cognitive des images de la ville : Evolution de la représentation cognitive de Paris auprès d'étudiants étrangers, thèse de psychologie, Université Paris-Descartes.

Ramadier T. (2004). Transdisciplinarity and its challenges: The case of urban studies, Futures, vol. 36, nº 4, p. 423-439.

Ramadier T. (2009). Capital culturel, lisibilité sociale de l'espace urbain et mobilité quotidienne, In Françoise Dureau et MarieAntoinette Hily (Eds.), Les mondes de la mobilité, Presses Universitaires de Rennes, p. 137-160.

Ramadier T., Moser G. (1998). Social legibility, the cognitive map and urban behaviour, Journal of Environmental Psychology, vol. $18, n^{\circ} 3$, p. 307-319.

Ramadier T., Petropoulou C., Bronner A-C., Borja S. (2008). Les usages paysagers de la ville : le paysage urbain comme régulateur des mobilités quotidiennes, In J-C. Wieber et T. Brossard (dir.), Paysage et information géographique, Edition Lavoisier-Hermès Science, p. 287-312.

Ramadier T. (2002). Rapport au quartier, représentation de l'espace et mobilité quotidienne, Espace et Société, n 108-109, p. 111-131.

Remy J. (2015). L'espace, un objet central de la sociologie, Toulouse, Érès.

Rivière C. (2017). La fabrique des dispositions urbaines. Propriétés sociales des parents et socialisation urbaine des enfants, Actes de la recherche en sciences sociales, $\mathrm{n}^{\circ} 216-217$, p. 64-79

Saarinen T (1987). Centering of mental maps of the world, discussion paper, department of geography and regional development, Tucson, Arizona

Sadalla E., Burroughs, W., Staplin, L. (1980). Reference points in spatial cognition, Journal of Experimental Psychology: Human Learning and Memory, vol. 6, n5, p. 516-528.

Shepard R. (2001). Perceptual-cognitive universals as reflections of the world, Behavioral and Brain Sciences, vol. 24, $\mathrm{n}^{\circ} 4, \mathrm{p}$. 581-601.

Steven A., Coupe P. (1978). Distortions in juged spatial relations, Cognitive Psychology, n¹0, p. 422-437.

Vidal De La Blache P. (1902). Les conditions géographiques des faits sociaux, annales de géographie, ${ }^{\circ} 11$, p. 13-23.

Witkin H. A. (1949). Perception of body position and of the position of the visual field, Psychological Monographs, $\mathrm{n}^{\circ} 63, \mathrm{p}$. $1-46$. 